\title{
Surfaces
}

\section{In Im-Memoriam, Bill Readings}

\section{Jean-François Vallée}

Volume 6, 1996

LES ÉCONOMIES DISCURSIVES DU SAVOIR ET DE LA CULTURE DANS

LE SILLAGE DE L'OEUVRE DE BILL READINGS

THE DISCURSIVE ECONOMIES OF KNOWLEDGE AND CULTURE,

WITH CONSTANT REFERENCE TO THE WORK OF BILL READINGS

URI : https://id.erudit.org/iderudit/1064846ar

DOI : https://doi.org/10.7202/1064846ar

Aller au sommaire du numéro

Éditeur(s)

Les Presses de l’Université de Montréal

ISSN

1188-2492 (imprimé)

1200-5320 (numérique)

Découvrir la revue

Citer ce document

Vallée, J.-F. (1996). In Im-Memoriam, Bill Readings. Surfaces, 6.

https://doi.org/10.7202/1064846ar d'utilisation que vous pouvez consulter en ligne. 


\section{Préface}

\section{In Im-Memoriam, Bill Readings}

Jean-François Vallée

Université de Montréal

Surfaces Vol. VI. 201a (V.1.0F - 15/11/1996) - ISSN:

1188-2492

Tout texte reste la propriété de son auteur. Néanmoins, Surfaces demande d'être citée à l'occasion de toute autre publication du texte en question.

\section{Une préface en forme d'appel}

THIS is what you need to do..., commençait Bill Readings lorsqu'il décidait de prendre votre destin en main. Ce qui lui arrivait assez fréquemment. Du moins, dès qu'il sentait un doute, une angoisse, ou du découragement chez son interlocuteur ou son interlocutrice. Un interventionnisme aussi généreux - - et aussi assuré - ne constitue certainement pas la norme chez les gens pressés que l'on trouve aujourd'hui dans nos excellentes institutions académiques. Ce souci est devenu encore plus rare. L'absence de Bill se fait cruellement sentir, ici à la revue Surfaces, au Département de littérature comparée, à Montréal, mais, aussi, sans aucun doute, dans la vie de nombre de ses amis et collègues un peu partout à travers le monde.

Dieu sait, par exemple, que j'aurais eu besoin de ses conseils - et de ses talents de lecteur et de rhéteur - - au moment de rédiger cette préface. C'est pourquoi il m'importe d'abord de saluer le courage des dix-sept auteurs qui ont répondu à notre premier appel d'articles. Non seulement devaient-ils travailler seuls, surmonter l'absence douloureuse du collègue, de l'ami, mais ils devaient aussi écrire moins d'un an après le choc, alors 
que la blessure était toujours vive. Écrire un texte dédié à Bill impliquait, dans un premier temps, d'admettre l'inadmissible : qu'il nous avait bel et bien quitté beaucoup trop tôt, beaucoup trop brutalement - en ce 31 octobre 1994. Une date qui, pour beaucoup d'entre nous, marque un événement inassimilable, une césure dans l'espace-temps de nos vies.

On ne peut s'empêcher de songer à l' événementialité de l'événement telle que la définissait Bill lui-même à la suite de Lyotard : "the fact or case that something happens, after which nothing will be the same again."[ $\underline{\mathbf{1}}$ ] Mais il reste que, comme le notait Andrew Benjamin lors d'une cérémonie tenue à Balliol College,[ $\underline{\mathbf{2}}$ ] on ne doit pas penser un tel événement sur le mode du mémorial, ou encore de la monumentalisation. Pour être vraiment fidèle à la mémoire de notre ami et collègue, la notion lyotardienne d'immémorial paraît plus appropriée. Bill définissait ainsi cette notion dans Introducing Lyotard: "That which can neither be remembered (represented to consciousness) nor forgotten (consigned to oblivion). It is that which returns, uncannily."[ $\underline{\mathbf{3}}$ ] Ainsi, si nous avons une tâche suite à l'événement que constitue le décès de Bill Readings, c'est d'assurer ce processus d'"immémorialisation", de favoriser l'oscillation entre le refus de l'oubli et le refus du simple souvenir représentatif, non problématique : "The task of not forgetting, of anamnesis, (...) which struggles to keep events from sinking into the oblivion of either representation (voice) or silence."[ $\underline{4}]$

Ce numéro spécial de la revue Surfaces veut s'insérer dans cet espace paradoxal, entre la voix et le silence, entre l'oubli et la mémoire, dans un processus d'anamnèse qui ne prétend certainement pas représenter la pensée, l'oeuvre, et encore moins la personne, de Bill Readings, mais qui incite aussi à ne pas sombrer dans le silence. Il importait de refuser d'accepter cet événement comme faisant partie d'un passé révolu, de l'ordre des choses, ce qui reviendrait à en faire un non-événement.

Plus encore, il importait de ne pas perdre - et parfois de reprendre - le fil des nombreuses conversations et tout aussi multiples débats qu'avait entamés, ou dans lesquels était intervenu, Bill Readings. Cette collectivité de textes se veut donc, avant tout, une célébration non seulement de notre amitié ou de notre respect pour Bill, mais surtout de la manière qu'il avait de concevoir le travail de la pensée : comme un travail à élaborer en communauté. Sauf que cette communauté ne doit pas 
prétendre à l'harmonie consensuelle. Il doit s'agir d'une communauté du dissensus - dans l'esprit de JeanFrançois Lyotard - dans laquelle, au nom de la justice, on ne saurait tenter de réduire les différends. Dans un très beau passage de The University in Ruins, Bill définit d'ailleurs plus précisément le sens qu'il voulait accorder à celle-ci :

Such a community, the community of dissensus that presupposes nothing in common, would not be dedicated either to the project of a full understanding (autonomy) or to a communicational consensus as to the nature of its unity. Rather, it would seek to make its heteronomy, its differences, more complex. To put this another way, such a community would have to be understood on the model of dependency rather than emancipation. We are, bluntly speaking, addicted to others, and no amount of twelve-stepping will allow us to overcome that dependency, to make it the object of a fully autonomous subjective consciousness. The social bond is thus a name for the incalculable attention that the heteronomous instance of the Other (the fact of others) demands. There is no freeing ourselves from the sense of the social bond, precisely because we do not come to the end of it; we can never totally know, finally and exhaustively judge, the others to which we are bound. Hence we cannot emancipate ourselves from our dependency on others.[ $\underline{\mathbf{5}}$ ]

Ainsi, c'est dans cet esprit de dépendance nonconsensuelle aux autres que nous voulons présenter ce numéro spécial de la revue Surfaces. Il faut cependant admettre qu'à l'origine de ce projet, et pour éviter surtout la collection d'essais à la manière du Festschrift qui risque toujours de prendre la forme d'un hommage figé et éclectique des survivants au maître ou à l'ami disparu -, Terry Cochran et moi-même avions choisi de proposer un thème relativement précis. Nous voulions faire de cette entreprise de publication non pas un rassemblement cacophonique de monologues, mais un lieu de conversation animée comme les aimait Bill. Le sujet ainsi que le titre que nous avions choisis - Les

économies discursives du savoir, dans le sillage de l'oeuvre de Bill Readings - se voulaient suffisamment vagues pour permettre divers types d'interventions tout en évitant autant que possible le méli-mélo habituel des recueils de textes en hommage à . 
Il en a résulté non pas un discours homogène sur le thème que nous avions proposé, mais un groupe relativement hétérogène d'interventions qui font référence selon différentes modalités tant au sujet proposé qu'à divers aspects de l'oeuvre, de la pensée voire même de la vie de Bill Readings. Le caractère fort hétéronome de cette pensée et son refus des consensus faciles auraient dû nous rendre plus prudents dans nos espoirs d'harmonie éditoriale. Bill aura refusé de nous laisser réduire son héritage intellectuel à un consensus illusoire, et nous aura ramené - un peu malgré nous - à la réalité vivifiante, mais parfois dérangeante, de la communauté du dissensus .

Ceci dit, nous avons tout de même résisté quelque peu aux forces centrifuges. À défaut d'harmonie ou de consensus réels, nous avons tenté - en éditeurs consciencieux - d'accorder une certaine cohérence à l'ensemble. Voilà pourquoi nous avons choisi de regrouper les dix-sept interventions ici réunies autour de quelques noyaux thématiques représentant certaines des principales lignes de force de la pensée et du travail de Bill Readings.

Mais d'abord, étant donnée l'importance qu'ont eu la pensée et l'oeuvre de Jean-François Lyotard dans le travail de Bill Readings, [ $\underline{\mathbf{6}}$ ] nous avons décidé de placer en exergue le texte inédit que celui-ci - malgré sa santé défaillante et son horaire extrêmement chargé l'année dernière - nous a diligemment fait parvenir. Il s'agit du texte remanié d'une conférence - - donnée à Tokyo il y a quelques années - qui concerne notamment le concept de postmodernité après la Condition postmoderne, et en rapport plus particulièrement avec l'histoire de la musique occidentale. Bill, un mélomane averti aux goûts éclectiques, aurait sûrement apprécié le fait que ce numéro spécial débute sur une note musicale.

Nous avons ensuite constitué quatre noyaux thématiques qui regroupent des textes partageant des préoccupations relativement similaires. Ce genre de découpage implique toujours une part d'arbitraire. Il aurait fallu pour mieux témoigner de l'hétérogénéité tant interne qu'externe des articles de ce numéro établir une sorte de configuration hypertextuelle et rhizomatique que nous avons préféré laisser à d'autres, pour l'instant, au profit d'une classification plus conventionnelle.

Le premier noyau thématique - intitulé L'université, audelà des ruines - regroupe cinq articles qui abordent sous divers angles le statut précaire ainsi que divers 
aspects problématiques de cette institution qui a constitué, comme on le sait, l'objet des réflexions ultimes de Bill Readings. Des réflexions que le destin - appuyé par le zèle éditorial courageux de Diane Elam - a eu la délicatesse de laisser s'incarner sous la forme posthume de The University in Ruins. On trouvera ici des essais, écrits pour la plupart avant la parution de The University in Ruins, mais qui abordent la question de l'université en s'inspirant tout de même fréquemment des réflexions sur le sujet préalablement publiées par Bill Readings.[ 7 ] D'abord J. Hillis Miller, dans Studying English Literature in the Transnational University, s'interroge sur l'avenir de la discipline des études littéraires - et plus généralement, des humanities - dans le contexte déstabilisant de la mondialisation, de la nature de plus en plus corporative des universités ainsi que du développement des nouvelles technologies de l'information et de la communication. Dans Memoir: In Celebration of Academic and Athletic Excellence, Steve Melville adopte une approche plus autobiographique (et sportive !) pour réfléchir - à partir de l'exemple de l'université de Syracuse où il a enseigné avec Bill Readings - sur divers enjeux politiques et éthiques qui se font jour dans une communauté académique. Jean-Ernest Joos, dans Politiques de l'autorité. Le pouvoir de celui qui n'est rien, part d'un point de vue plus philosophique pour remettre en cause la figure moderne - invisible et soi-disant neutre - de la transmission de l'autorité académique afin de proposer un type de configuration habité par la matérialité et la corporalité de l'enseignant (en prenant exemple notamment sur les programmmes d'études spécifiques : études gaies, féministes, afro-américaines, etc.). Dans Transforming Differences into "Normality" - German Unification and the Crisis of the Humanities, Karlheinz Barck nous fait traverser l'Atlantique pour décrire les problèmes survenus lors de la réunification du système universitaire allemand qui a mené à une normalisation et une dépolitisation des sciences humaines inspirées, pour le meilleur mais aussi pour le pire, par le modèle occidental. Enfin, dans The Predicament of Culture at Room Temperature, Jennifer Allen s'attaque aux enjeux et aux transformations actuels de la disciplinarité par le biais d'une réflexion sur le cas problématique des Cultural Studies.

Le second noyau thématique - intitulé Politiques, éthiques, épistémiques - regroupe quatre textes fort différents, mais qui s'adressent tous à diverses préoccupations centrales de la pensée politique et éthique de Bill Readings. S'y croisent notamment les notions d'événement, d' impertinence politique, de 
vitesse, de temporalité, etc. En premier lieu, dans Sifflements dans l'obscurité. L'événementialité de Bill Readings, Johanne Villeneuve entame un dialogue critique serré avec la pensée de Bill Readings, en ce qui concerne surtout la notion d'événementialité ainsi que ses enjeux éthiques et épistémiques. Dans Just Politics: Bill Readings' Impertinent Call, Valeria Wagner - à partir de réflexions de Bill Readings sur le politique et d'exemples concrets - aborde de front les difficiles questions d'une politique juste ("just politics") et du degré d' impertinence nécessaire pour résister à la tentation universaliste du discours moderne sans paralyser pour autant l'efficience de l'action politique. Dans In Memoriam, Fast Forward, Marc Redfield s'attarde sur la question de la temporalité postmoderne décrite par Bill Readings, et plus particulièrement sur la notion de vitesse qui précède toute représentation et conceptualisation. Dans Answering the Question: What is an Intellectual?, Gary Hall pose plus généralement la question toujours actuelle du statut de l'intellectuel d'hier à aujourd'hui. Enfin, Germain Lacasse, dans L'aura du futur antérieur. La postmodernité et la fin de l'histoire, trace - au futur antérieur - un portrait de la notion de postmodernité avancée par Bill Readings, et ce plus particulièrement à travers les notions de temps filmique et cinématographique.

Le troisième noyau thématique - Éthiques de la lecture - nous ramène à un autre thème fondamental de l'oeuvre et de la pensée de Bill Readings : la lecture. Cette question paraissait incontournable même si l'on voulait éviter la tentation des mauvais jeux de mots sur le patronyme de Bill. Dans Dette, institution et histoire, Éric Méchoulan tente de régler des comptes avec la question de la dette à travers des réflexions interreliées sur l'institution, le discours historiographique, l'altérité et les morts. En exergue se trouve d'ailleurs une citation de Bill Readings qui parle d'elle-même : "Reading is not a matter of mimetic representation or conceptual critique: it is an ethical practice."[ $\mathbf{8}$ ] Comme pour démontrer la pertinence de cette définition, Marie Lessard, dans Squattage et terrains vagues, tente de repenser les pratiques d'écriture de l'autobiographie féminine contemporaine (notamment chez Marguerite Duras et Kathy Acker) à partir de la notion féconde du squattage . Enfin, dans Rhapsodic Readings, à travers une lecture serrée de l'Ion de Platon suivie d'un bond historique du côté de Sainte-Beuve et de T. S. Eliot, Craig Moyes pose la question du statut problématique de la connaissance littéraire que proposent notamment professeurs et théoriciens de la littérature aujourd'hui. 
Le quatrième, et dernier, noyau thématique - Re-

lectures de la Renaissance - nous ramène aux sources du travail de Bill Readings qui avait, comme on le sait, rédigé sa thèse sur Milton et Marvell.[ $\underline{\mathbf{9}}$ ] Dans Shakespeare and the Formation of the Modern Economy, Richard Waswo montre les rapports étroits qui se tissent entre argent et langage alors que Shakespeare met en scène les principes de l'économie moderne. Puis, Marshall Grossman, dans Subsequent Precedence. Milton's Materialistic Reading of Ficino and Tasso, part d'une remarque prémonitoire de Bill Readings afin d'entreprendre une lecture sophistiquée de Milton qui met en lumière les complexités de la temporalité métaleptique des discours de la rhétorique prophétique et du poétique. Enfin, Beatrice Skordili, dans Bill Readings and the Supplement, s'essaie au genre de la grapho-biographie en tirant du travail de Bill Readings sur Milton des réflexions sur son éthique de la lecture, de l'écriture et de la pédagogie.

Tous les articles précédents tracent un parcours intellectuel fort impressionant inspiré en grande partie par la pensée, l'oeuvre voire même la vie de notre ami et collègue. Il va de soi cependant qu'à travers cette division quadripartite des articles de ce numéro, et malgré la richesse et la diversité des textes en présence, nous ne prétendons pas représenter toutes les facettes du travail de Bill Readings. D'autant plus que certaines questions, pourtant très présentes dans son oeuvre, ne trouvent pas d'écho significatif dans ce volume. À ce titre, on pourrait citer, pour ne donner qu'un exemple, les questions qui concernent l'histoire de l'art et le concept de la vision. On peut évidemment considérer de telles lacunes comme inévitables dans le contexte d'un simple numéro de revue savante. Mais on pourrait aussi tenter d'y rémédier...

En effet, cette revue savante s'adonne - manifestement - à être diffusée sous forme électronique. Il nous paraîtrait donc particulièrement approprié de profiter ici des potentialités et de la plasticité de cette nouvelle technologie de publication non seulement pour combler les quelques lacunes thématiques de ce numéro déjà fort riche, mais aussi parce que nous nous sommes rendus compte que beaucoup d'amis et de collègues de Bill, au moment de notre premier appel d'articles, se trouvaient dans l'impossibilité d'écrire quoi que ce soit. De plus, audelà de ces préoccupations d'éditeurs généreux, il nous apparaîtrait comme particulièrement approprié de mettre à profit les possibilités de l'édition électronique 
pour créer ici une entité organique et en croissance perpétuelle dans le contexte d'une publication justement dédiée à quelqu'un d'aussi peu "statique" que Bill Readings.

À notre déjà impressionnant groupe de dix-sept auteurs, nous souhaiterions donc en ajouter d'autres qui leur répondraient, qui s'adresseraient à nouveau au travail de Bill Readings ou encore qui ajouteraient leur propre contribution au processus d'anamnèse, à cette conversation qui refuserait ainsi la clôture, et, plus encore, la forclusion. Ainsi, notre communauté d'auteurs, et de lecteurs, - fût-elle virtuelle - tendra asymptotiquement vers cette fameuse communauté du dissensus qui accepte d'entrer dans le processus inachevable de la dépendance envers l'Autre, les autres...

De nouveaux textes, des témoignages, des compte rendus, des débats pourront dorénavant se greffer à ce volume dans les mois, voire les années qui viennent. Ces nouvelles interventions pourront se joindre, par la magie des liens hypertextuels, à l'un ou l'autre des noyaux thématiques déjà créés dans ce numéro ou bien ils pourront être à la source de nouveaux noyaux. On peut même imaginer, à plus long terme, la création de liens hypertextuels à l'intérieur des textes pour relier, très concrètement, les articles qui se feront écho. Mais les modalités de croissance de ce volume rhizomatique de Surfaces restent encore - en partie du moins - à déterminer. En fait, la forme que prendra la suite de ce volume dépend autant de vous que de nous.

Nous - et vous - voilà donc revenus aux points de suspension de l'incipit typiquement readingsien de cette préface : "This is what you need to do..."

\section{NOTES}

1. Bill Readings, Introducing Lyotard. Art and Politics, Routledge, 1991, p. xxxi.

2. Cette cérémonie a eu lieu au mois de novembre 1994 au Balliol College de l'université d'Oxford.

3. Introducing Lyotard, op. cit., p.xxxii. 
4. Ibid.

5. Bill Readings, The University in Ruins, Harvard University Press, 1996.

6. Outre le déjà cité Introducing Lyotard publié chez Routledge, Bill Readings a édité et traduit les écrits politiques de Lyotard (Political Writings, Bill Readings éd., trad. Bill Readings et Kevin Paul Geiman, University of Minnesota Press, 1993.). Les références à la pensée lyotardienne émaillent aussi une bonne partie de ses autres publications.

7. "From Emancipation to Obligation: Sketch for a Heteronomous Politics of Education." (Education and the Postmodern Condition. Michael Peters éd., Auckland \& New York: Bergin and Garvey, 1994); "Be Excellent : Culture, the State, and the Posthistorical University." (Alphabet City 3, numéro spécial sur "The States of Culture", 1993); "Identity Crisis: The University and Culture." (ACCUTE Newsletter, 1993).

8. Bill Readings, Introducing Lyotard. Art and Politics, Routledge, 1991.

9. Bill Readings, The Restoration and the Fall of Language: The Search for Meaning in the Poetry of Marvell and Milton, D. Phil. thesis. University of Oxford, 1985.

Accueil Surfaces | Table des matières | Recherche Surfaces Home Page | Table of Contents | Search

PUM | Livres | Revues | Publications électroniques | Vente et distribution 